\title{
Ultrasound screening for cholangiocarcinoma could detect premalignant lesions and early-stage diseases with survival benefits: a population-based prospective study of 4,225 subjects in an endemic area
}

Prakongboon Sungkasubun ${ }^{1}$, Surachate Siripongsakun ${ }^{1}$, Kunlayanee Akkarachinorate², Sirachat Vidhyarkorn ${ }^{1}$, Akeanong Worakitsitisatorn', Thaniya Sricharunrat ${ }^{1}$, Sutida Singharuksa', Rawisak Chanwat ${ }^{4}$, Chairat Bunchaliew$^{4}$, Sirima Charoenphattharaphesat ${ }^{1}$, Ruechuta Molek', Maneenop Yimyaem', Gaidganok Sornsamdang ${ }^{1}$, Kamonwan Soonklang ${ }^{1}$, Kasiruck Wittayasak', Chirayu U. Auewarakul ${ }^{1,3}$ and Chulabhorn Mahidol 1,3,5*

\begin{abstract}
Background: Thailand has a high incidence of cholangiocarcinoma (CCA), particularly in the north and northeastern regions. Most CCA patients come at a late, unresectable stage and presently no optimal screening test for CCA has been established. We determined the prevalence of CCA in a remote northern village and explored if screening could lead to early detection and survival benefits.

Methods: A 5-year population-based study was started in October, 2011 for consented Thai individuals, aged 30-60 years. The screening program comprised blood testing, stool examination and serial ultrasonography every 6 months.

Results: During the first 3 years, 4,225 eligible individuals were enrolled. CCA was detected in 32 patients, with a mean age of 51.9 years (41-62 years), and 21/32 cases were at a curative resectable stage. The prevalence rate of CCA was 165.7 per 100,000 and one- and two-year incidence rate was 236.7/100,000 and 520.7/100,000, respectively. One- and 2-year overall survival rates of CCA patients were 90.9 and $61.5 \%$, respectively. Prognosis was better in resectable cases with $100 \%$ 1-year and $77.8 \%$ 2-year survival rates. Interestingly, premalignant pathological lesions (stage 0) were identified in 11 cases with $100 \% 3$-year survival rate. Serum biomarkers and alkaline phosphatase were not sufficient to detect early-stage disease. In 22 patients, stool samples were positive for Opisthorchis viverrini, based on polymerase chain reaction.
\end{abstract}

Conclusion: Detection of premalignant lesions and early-stage resectable CCA by ultrasonography resulted in improved clinical outcome. Ultrasonography should be offered as a first screening tool for CCA in an endemic area until other useful biological markers become available.

Keywords: Cholangiocarcinoma, Premalignant lesions, Cancer screening, Early detection, Ultrasonography, Tumor markers

\footnotetext{
* Correspondence: cmah2500@gmail.com

${ }^{1}$ Chulabhorn Hospital, 54 Kamphaeng Phet 6 Road, Laksi, Bangkok 10210,

Thailand

${ }^{3}$ Faculty of Medicine Siriraj Hospital, Mahidol University, 2 Wanglang Road,

Bangkoknoi, Bangkok 10700, Thailand

Full list of author information is available at the end of the article
} 


\section{Background}

Cholangiocarcinoma is a tumor of the biliary tract, presumably of cholangiocytic origin, with a rising global incidence [1-6]. Several known risk factors exist, linking chronic biliary inflammation to the pathogenesis of cholangiocarcinoma [7, 8]. Late presentation to hospital, with a median survival of months, is noted in most patients in developing countries whereby cholangiocarcinoma is most prevalent. Moreover, the pathological or cytological diagnosis of cholangiocarcinoma is not always accessible despite indications from imaging studies and clinical condition [7-10]. Surgical resection is the current therapy of choice for every type of cholangiocarcinoma $[8,11,12]$. Resection offers the best opportunity for long-term survival. Nevertheless, in the minority of patients and in those with large node-positive or multifocal intrahepatic cholangiocarcinoma, resection seems to provide little benefit $[10,12]$. Overall, the 5-year survival in cholangiocarcinoma cases is poor, with $60 \%$ to $>90 \%$ recurrence rates $[7,9,13]$.

Cholangiocarcinoma is relatively rare in most western countries, but high incidence rates have been reported in Eastern Asia, especially in Thailand [5, 14-16]. The etiology of this cancer appears to be mostly due to specific infectious agents $[17,18]$. In 2009, infections with liver flukes, Clonorchis sinensis or Opisthorchis viverrini, were both classified as carcinogenic to humans by the International Agency for Research on Cancer for cholangiocarcinoma $[14,18]$. With the current systematic tumor registration in Thailand, new high-incidence areas have been identified in North and Northeastern Thailand $[10,17,19]$. Ban Luang is a district in the western part of Nan Province in Northern Thailand and is divided into 4 sub-districts. Based on a previous study, the incidence of liver cancer in Ban Luang was 138.8 per 100,000 persons, which is higher than that of other regions of the world or even in Khon Kaen Province, which is previously reported as an endemic area for cholangiocarcinoma [17, 20, 21].

The present study aimed to ascertain the prevalence and incidence of cholangiocarcinoma, to identify predisposing factors, and to explore whether screening could lead to early treatment and reduction of morbidity and mortality rates of cholangiocarcinoma patients.

\section{Methods}

\section{Study design and population}

A population-based, prospective cohort study for cholangiocarcinoma screening included liver ultrasonography, stool examination for parasites, complete blood count (CBC), liver function tests (LFT) including alkaline phosphatase (ALP), and measurements of hepatitis B surfaceantigen, hepatitis B core antibody, and serum carcinoembryonic antigen (CEA), carbohydrate antigen (CA)19-9, and $\alpha$-fetoprotein (AFP) every 6 months from October 2011 to September 2016. This study was approved by the Ethics Committee for Human Research of Chulabhorn Research Institute, Bangkok, Thailand (Certificate no. 29/2554). Written informed consents were obtained from all the study participants.

Targeted subjects were all indigenous residents of Ban Luang District, aged 30-60 years, who were not pregnant, or breast feeding, or diagnosed with or under treatment for any type of cancer. Of 6,327 targeted subjects based on a district census registration, 4,337 consented to the study and were recruited by village health volunteers with the cooperation of Ban Luang Hospital. Natural history and prevalence and incidence rates of cholangiocarcinoma were investigated, along with an analysis of associated risk factors and a comparison of results between liver ultrasonography and laboratory testing. People with liver lesions suspected of liver cancer, such as isolated mass lesions, masses associated with bile duct dilatation, or isolated bile duct dilatation without mass lesions were referred for further imaging studies, including computed tomography (CT), magnetic resonance imaging (MRI) or magnetic resonance cholangiopancreatography (MRCP) at Chulabhorn Hospital. All cholangiocarcinoma treatments, that is, surgery, chemotherapy or radiotherapy, were performed at Chulabhorn Hospital.

\section{Laboratory and ultrasonography studies}

LFT and tumor markers were performed by Cobas 6000 (c501 and e601) of Roche Diagnostics (Thailand) and liver ultrasonography was performed using Logiq C2 ultrasound system (GE Healthcare) and Aplio 300 and 500 ultrasound system (Toshiba). Recommended diagnostic cut-off value for CA19-9, CEA and alkaline phosphatase (ALP) was $>37 \mathrm{U} / \mathrm{mL}, \geq 4.7 \mathrm{ng} / \mathrm{mL}$, and $>100 \mathrm{IU}$, respectively. Liver ultrasonography was performed by a team of radiologists from Chulabhorn Hospital and Nan Hospital. Criteria for further CT, MRI, and/or MRCP investigations included nodule/mass lesion, nodule/mass with bile duct dilatation, and focal bile duct dilatation. In case of diffuse bile duct dilatation without other associated abnormality, MRCP was performed to exclude small biliary intraductal lesions by using a peripheral bile duct diameter of $\geq 3 \mathrm{~mm}$. Patients who were diagnosed as having suspicious/definite malignant lesions by CT, MRI, and MRCP were subsequently reviewed by a multidisciplinary team for further treatment planning. All cancer specimens were pathologically diagnosed at Chulabhorn Hospital with routine hematoxylin and eosin $(H \& E)$ staining and immunohistochemistry was additionally performed if necessary. 


\section{Statistical analysis}

Demographic data were reported as mean and standard deviations for all continuous variables and as proportions and absolute counts for discrete variables. The Mann-Whitney $U$ test was used to compare continuous variables, whereas Pearson $\chi^{2}$ and Fisher's exact tests were used to compare discrete variables. A two-tailed $P$ $<0.05$ was considered to be significant to verify the assumptions for all statistical tests. Prevalence was calculated from cholangiocarcinoma patients detected by initial screening ultrasonography. Incidence was calculated from new cases detected by subsequent ultrasonographic studies. Disease-free survival (DFS) was defined as the length of time that the patient survived without any signs or symptoms, after primary treatment for cholangiocarcinoma was completed. Progression-free survival (PFS) was the length of time during and after treatment of cholangiocarcinoma that the patients lived with the disease, without deterioration or progression. Overall survival (OS) was the length of time that the patients were still alive, starting from the date of diagnosis or start of cholangiocarcinoma treatment.

\section{Results}

Demographic data of the cohort and prevalence and incidence of cholangiocarcinoma

Between October 2011 and April 2014, abdominal ultrasonography was completed in 4,225 participants $(1,919$ males and 2,306 females) from 4,337 recruited participants. Cholangiocarcinoma was detected in 32 patients, with a mean age of 51.9 years (41-62 years), comprising 18 men (56.3\%) and 14 women (43.7\%). Tables 1 and 2 shows a comparison between cholangiocarcinoma patients and non-cholangiocarcinoma population. There was no significant difference between cholangiocarcinoma patients and non-cholangiocarcinoma population regarding gender, smoking, history of parasitic infection and treatment, and raw freshwater animal consumption $(P>0.05)$. The mean age of cholangiocarcinoma patients was 51.9 years and that of non-cholangiocarcinoma cases was significantly lower at 45.7 years and alcohol consumption was significantly different between the 2 groups. History of unclassified liver cancer or cholangiocarcinoma in first-degree relatives was significantly higher in cholangiocarcinoma patients $(33.3 \%)$ than in the non-cholangiocarcinoma group (17.1\%).

Initial screening revealed 7 asymptomatic cases of cholangiocarcinoma among 4,225 participants. The prevalence rate of cholangiocarcinoma in the Ban Luang population aged 30-60 years was 165.7 per 100,000 . We subsequently detected $6,4,5,7$ and 3 cholangiocarcinomas from each 6-month follow-up period. The 1- and 2year incidence rates were $236.7 / 100,000(10 / 4,225)$ and $520.7 / 100,000(22 / 4,225)$, respectively.
Ultrasound findings, stages and resectability of cholangiocarcinoma patients

Of 32 cholangiocarcinoma patients, 10 showed masses associated with bile duct dilatation, 9 showed isolated mass lesions, 11 showed isolated bile duct dilatation, and the other 2 cases showed questionable liver masses with ultrasonography. Twenty-one cases were resectable and 11 unresectable. The most common type of cholangiocarcinoma was intrahepatic (21/32, $65.6 \%)$. Hilar type was found in 6 cases $(18.8 \%)$ and extrahepatic type in 5 cases (15.6\%). Based on AJCC Cancer Staging Manual, Seventh edition (2010) [22], there were 5, 10, 2, 2, 8 and 5 patients in stage I, II, IIIa, IIIb, IVa and IVb cholangiocarcinoma, respectively. In all stage I patients, resections were performed. In stage II disease, 9 patients were resected and 1 patient was medically inoperable. In stage IIIa, one patient was resected and the other was unresectable. Similarly, in stage IIIb, one patient was resected and the other was unresectable. In stage IVa, 5 patients had lymph node metastasis but still resectable lesions and 3 patients were unresectable. All patients in stage IVb were unresectable due to M1 disease. Additionally, we found 11 patients with premalignant lesions (or stage 0) (Table 3). With regards to false positive ultrasonography, we had 3 cases whose surgical specimen revealed no malignancy despite suspicious $\mathrm{CT}$ and MRI results. The pathological reports were chronic cholangitis with cirrhosis, adenoma with periductal fibrosis and calcified fibrotic cyst.

\section{Survival rates of patients with cholangiocarcinoma and premalignant lesions}

Over a follow-up period, 1- and 2-year survival rates were 90.9 and $61.5 \%$, respectively, for CCA cases (Table 4). In resectable cases, 1- and 2-year survival rates were $100 \%(16 / 16)$ and $77.8 \%$ (7/9). One- and two-year survival rates were lower in unresectable cases; $66.7 \%$ $(4 / 6)$ and $25 \%(1 / 4)$, respectively. In resectable cases, 1and 2-year DFS and recurrent free survival rates were $75 \%(12 / 16)$ and $44.4 \%$ (4/9), respectively. All patients with premalignant lesions had excellent outcomes after surgery (100\% OS and $100 \%$ 2-year DFS).

\section{Values of LFT, tumor markers and stool examination for parasites}

Serum biomarkers, CA19-9, CEA or ALP were analyzed among cases with and without cholangiocarcinoma as shown in Table 5. Sensitivity of CA19-9, CEA and ALP were $18.75,34.38$ and $50.00 \%$, respectively. When these tumor makers were combined, the sensitivity was still low (68.75 \%). Stool examination was performed in 3,663 individuals $(86.67 \%)$. There were 7 types of parasites in 824 cases $(22.50 \%)$. O. viverrini-like eggs were found in 710 cases (19.38\%), Taenia eggs in 56 cases 
Table 1 Demographic data of cholangiocarcinoma patients and non-cholangiocarcinoma population

\begin{tabular}{|c|c|c|c|c|}
\hline Demographic data & Total & $\begin{array}{l}\text { Cholangiocarcinoma } \\
\text { patients }(n=32)\end{array}$ & $\begin{array}{l}\text { Non- cholangiocarcinoma } \\
\text { population }(n=4,193)\end{array}$ & $P$ value \\
\hline Gender & 4,225 & 32 & 4,193 & $0.334^{a}$ \\
\hline \multirow[t]{2}{*}{ Male } & 1,919 & 18 & 1,901 & \\
\hline & $(45.4)$ & $(56.3)$ & $(45.3)$ & \\
\hline \multirow[t]{2}{*}{ Female } & 2,306 & 14 & 2,292 & \\
\hline & $(54.6)$ & $(43.7)$ & $(54.7)$ & \\
\hline Age (year) & 45.71 & 51.91 & 45.68 & $<0.001^{\mathrm{c}}$ \\
\hline History of smoking & 4,217 & 27 & 4,190 & $0.092^{\mathrm{a}}$ \\
\hline \multirow[t]{2}{*}{ Never } & 2,756 & 14 & 2,742 & \\
\hline & (65.4) & $(51.9)$ & $(65.4)$ & \\
\hline \multirow[t]{2}{*}{ Ex-smoker } & 718 & 9 & 709 & \\
\hline & $(17.0)$ & (33.3) & $(16.9)$ & \\
\hline \multirow[t]{2}{*}{ Active smoker } & 743 & 4 & 739 & \\
\hline & $(17.6)$ & $(14.8)$ & $(17.7)$ & \\
\hline Alcoholic consumption & 4,217 & 27 & 4,190 & $<0.001^{\mathrm{b}}$ \\
\hline \multirow[t]{2}{*}{ Never } & 1,315 & 9 & 1,306 & \\
\hline & $(31.2)$ & $(33.4)$ & $(31.2)$ & \\
\hline \multirow[t]{2}{*}{ Ex-consumption } & 492 & 10 & 482 & \\
\hline & $(11.7)$ & $(37.0)$ & $(11.5)$ & \\
\hline \multirow[t]{2}{*}{ Light consumption (< once a month) } & 1,360 & 4 & 1,356 & \\
\hline & $(32.2)$ & $(14.8)$ & $(32.3)$ & \\
\hline \multirow[t]{2}{*}{ Moderate to heavy consumption } & 1,050 & 4 & 1,046 & \\
\hline & $(24.9)$ & $(14.8)$ & $(25.0)$ & \\
\hline Any liver cancers or cholangiocarcinoma in first-degree relatives & 4,202 & 27 & 4,175 & $0.037^{\mathrm{a}}$ \\
\hline \multirow[t]{2}{*}{ Yes } & 722 & 9 & 713 & \\
\hline & $(17.2)$ & $(33.3)$ & $(17.1)$ & \\
\hline \multirow[t]{2}{*}{ No } & 3,480 & 18 & 3,462 & \\
\hline & (82.8) & $(66.7)$ & $(82.9)$ & \\
\hline Diagnosis of liver fluke & 4,153 & 27 & 4,126 & $0.366^{a}$ \\
\hline \multirow[t]{2}{*}{ Ever } & 753 & 7 & 746 & \\
\hline & $(18.1)$ & $(25.9)$ & (18.1) & \\
\hline \multirow[t]{2}{*}{ Never } & 3,293 & 19 & 3,274 & \\
\hline & (79.3) & (70.4) & (79.3) & \\
\hline \multirow[t]{2}{*}{ Unknown } & 107 & 1 & 106 & \\
\hline & (2.6) & (3.7) & (2.6) & \\
\hline
\end{tabular}

a Fisher's exact test

b Pearson's $X^{2}$ test

c Mann-Whitney $U$ test

(6.17\%), Sarcocystis spp. eggs in 41 cases (4.42\%), Strongyloides stercoralis eggs in 17 cases (1.85\%), hookworm eggs in 45 cases (1.23\%), Trichuris trichiura eggs in 5 cases $(0.14 \%)$, and Enterobius vermicularis eggs in 2 cases $(0.05 \%)$. In 22 of 32 cholagiocarcinoma patients, stool samples were positive for Opisthorchis viverrini, based on polymerase chain reaction.

\section{Discussion}

Cholangiocarcinoma is a silent malignancy with a significantly high morbidity and mortality. Patients usually present at a late stage, rendering curative measures impossible to be performed to prolong lives [9, 13]. Although the annual incidence rate of cholangiocarcinoma in the western countries is low at $1-2$ cases per 100,000 , 
Table 2 Demographic data of cholangiocarcinoma patients and non-cholangiocarcinoma population

\begin{tabular}{llll}
\hline Demographic data & Total & $\begin{array}{l}\text { Cholangiocarcinoma } \\
\text { patients (32 cases) }\end{array}$ & $\begin{array}{l}\text { Non- cholangiocarcinoma } \\
\text { population }(4,193 \text { cases) }\end{array}$ \\
\hline $\begin{array}{l}\text { Any treatment for liver flukes from physicians/public } \\
\text { health personnel }\end{array}$ & 4,129 & 27 & 4,102 \\
$\quad$ Ever & 1,441 & 7 & 1,434 \\
& $(34.9)$ & $(25.9)$ & $(35.0)$ \\
Never & 2,576 & 18 & 2,558 \\
& $(62.4)$ & $(66.7)$ & $(62.3)$ \\
Unknown & 112 & 2 & 110 \\
& $(2.7)$ & $(7.4)$ & $(2.7)$ \\
Raw freshwater fish/shrimp/snail consumption & 4,218 & 27 & 4,191 \\
Yes & 526 & 2 & 524 \\
& $(12.5)$ & $(7.4)$ & $(12.5)$ \\
No & 3,692 & 25 & 3,667 \\
& $(87.5)$ & $(92.6)$ & $(87.5)$ \\
\hline
\end{tabular}

a Fisher's exact test

b Pearson's $x^{2}$ test

c Mann-Whitney $U$ test

many studies have documented a steady increase in the incidence of intrahepatic cholangiocarcinoma over the past few decades; increases have been seen in North America, Europe, Asia, and Australia [2, 4, 6]. Our study confirmed a high prevalence and high incidence rate of cholangiocarcinoma in Thailand, particularly in the northern region of the country with a prevalence rate of 165.7 per 100,000 and 1 - and 2-year incidence rates of $236.7 / 100,000$ and 520.7/100,000, respectively [10, 17].

As a screening program for cholangiocarcinoma is currently not established worldwide, this study was intended to explore if abdominal ultrasonography or tumor markers could be of benefit to detect early-stage cases for curative intent in an endemic area. By performing successive ultrasonography every 6 months, we managed to identify 32 cholangiocarcinoma cases from 1 to 6 sessions over a 3-year period and $65 \%$ of our patients

Table 3 Pathological data of patients with premalignant lesions

\begin{tabular}{llll}
\hline No. & Sex & Age & Pathology \\
\hline 1 & M & 45 & Intraductal cholangiocarcinoma \\
2 & M & 60 & Intraductal papillary biliary neoplasm \\
3 & F & 53 & Biliary epithelial neoplasm \\
4 & F & 51 & Low grade dysplastic epithelium \\
5 & F & 52 & Intraductal papillary biliary neoplasm \\
6 & M & 41 & High grade dysplastic epithelium \\
7 & F & 59 & Hyperplasia + dysplastic epithelium \\
8 & F & 59 & Intraductal papillary biliary neoplasm \\
9 & F & 53 & Atypical bile duct epithelium \\
10 & F & 56 & Intraductal papillary biliary neoplasm \\
11 & M & 51 & Intraductal papillary biliary neoplasm \\
\hline
\end{tabular}

were diagnosed with early-stage and operable tumors which was markedly different from all the hospital-based data previously reported in Thailand whereby most cholangiocarcinoma cases presented at a late stage. A recent study from the National Cancer Institute of Thailand showed that there were $2.17,12.69,24.77$, and $57.89 \%$, respectively, of stage I, II, III, and IV of newly diagnosed cholangiocarcinoma cases diagnosed at their institute in 2013 [23]. Nevertheless, the most common tumor location in this study was intrahepatic which was similar to other published hospital-based studies [7, 12, 24].

Over a follow-up period of 3 years, 1 - and 2-year survival rates of the affected cases were high (>60\%), particularly in resectable cases (100 and $78 \%$, respectively) as expected, and were much better than those reported from previous studies in Thailand and Malaysia with advanced and inoperable cases [10, 11, 25]. Outcome data from a study in Khon Kaen of 411 intrahepatic cholangiocarcinoma patients revealed that only 138 cases were resectable and the mean survival time was 1,039 days in tumor stage III, 773 days in stage IVa, and 382 days in stage IVb [10]. Rare long-term survival with a oneyear survival of just $15 \%$ was reported in perihilar

Table 4 Survival outcomes of patients with premalignant lesions and cholangiocarcinoma

\begin{tabular}{lllllll}
\hline Diagnosis & \multicolumn{2}{l}{ Patients with } & \multicolumn{3}{c}{ Cholangiocarcinoma patients } \\
\cline { 3 - 7 } & Premalignant lesions & & \multicolumn{2}{l}{ Resectable } & \multicolumn{2}{c}{ Unresectable } \\
\hline \multirow{2}{*}{ OS } & 1 -year & 2-year & 1-year & 2-year & 1-year & 2-year \\
DFS/PFS & $100 \%$ & $100 \%$ & $75 \%$ & $44.4 \%$ & $33.3 \%$ & $0 \%$ \\
\hline
\end{tabular}

Abbreviations: OS overall survival, PFS progression free survival, DFS disease free survival 
Table 5 Tumor marker analysis in the cases with and without cholangiocarcinoma

\begin{tabular}{|c|c|c|c|c|c|c|c|}
\hline \multirow[t]{2}{*}{ Predictor } & \multicolumn{2}{|c|}{ Cholangiocarcinoma } & \multirow[t]{2}{*}{ Total } & \multirow[t]{2}{*}{ Sensitivity } & \multirow[t]{2}{*}{ Specificity } & \multirow[t]{2}{*}{ PPV } & \multirow[t]{2}{*}{ NPV } \\
\hline & Positive & Negative & & & & & \\
\hline Positive CA19-9 & 6 & 59 & 65 & 18.75 & 98.63 & 9.23 & 99.39 \\
\hline Negative CA19-9 & 26 & 4245 & 4271 & & & & \\
\hline Total & 32 & 4304 & 4336 & & & & \\
\hline Positive CEA & 11 & 749 & 760 & 34.38 & 82.60 & 1.45 & 99.41 \\
\hline Negative CEA & 21 & 3555 & 3576 & & & & \\
\hline Total & 32 & 4304 & 4336 & & & & \\
\hline Positive ALP & 15 & 641 & 656 & 50.00 & 83.54 & 2.29 & 99.54 \\
\hline Negative ALP & 15 & 3254 & 3269 & & & & \\
\hline Total & 30 & 3895 & 3925 & & & & \\
\hline Positive CA19-9 or CEA or ALP & 22 & 1252 & 1274 & 68.75 & 70.91 & 1.73 & 99.67 \\
\hline Negative CA19-9 or CEA or ALP & 10 & 3052 & 3062 & & & & \\
\hline Total & 32 & 4304 & 4336 & & & & \\
\hline
\end{tabular}

Abbreviations: CA19-9 carbohydrate antigen 19-9, CEA carcinoembryonic antigen, ALP alkaline phosphatase, NPV negative predictive value, PPV positive predictive value

cholangiocarcinoma cases [11]. Another long term follow-up data from Malaysia in 69 cholangiocarcinoma patients showed that the overall median survival was 4 months with the median survival of 16 months in R0 resected patients [25]. Overall 1-, 2and 3-year survival rates were 67, 17 and $17 \%$, respectively. In one study from the US, $53.8 \%$ of newly diagnosed intrahepatic cholangiocarcinoma patients were not candidates for resection [12]. In resectable groups, the median disease-specific survival was 36 months and recurrence was observed in most of the patients $(62.2 \%)$. In unresectable groups, the median survival varied depending on modalities of treatments, i.e., 22 months and 9 months in patients receiving regional chemotherapy as part of treatment and systemic chemotherapy alone, respectively. Although our outcomes could not be directly compared because other reports were from hospital-based studies, it is evident that screening ultrasonography could identify patients at an early and asymptomatic stage leading to better treatment outcomes.

Interestingly, our study identified 11 patients with premalignant lesions (stage 0 ) such as biliary intraepithelial neoplasia, intraductal papillary biliary neoplasm or dysplastic epithelium [26]. Our pathological results were achievable because we detected patients at the earliest stage whereas most studies of cholangiocarcinoma did not have pathological tissue due to the inoperative nature of the late-stage cases. These premalignant lesions represent cases whereby abnormal cells were found in the innermost layer of the tissue lining of the extrahepatic bile duct. All of them did better than the earlystage cholangiocarcinoma cases with $100 \%$ DFS and are still alive at 3-year follow-ups. Therefore, patients detected at a premalignant stage appeared to benefit the most from screening ultrasonography and potentially are cured.

With regards to demographic data, the population in Ban Luang is socioeconomically comparable to rural northern and northeastern population of Thailand with similar ethnic backgrounds [27]. The villagers' major occupation was farming with an average income per month of 1,000-4,999 THB (about 30-145 USD). The mean age of cholangiocarcinoma cases of 51.9 years old in this study was consistent with the average age of cholangiocarcinoma cases reported from the National Cancer Registry or hospital-based studies [28] but was much lower than most western data whereby most patients are in their 60 s or 70 s $[2,4,13]$. During our 3-year study period, no cancer patients under the age of 40 years old were identified. Hence, age could be another factor in disease development and cholangiocarcinoma screening in individuals younger than 40 years old may not be of value or necessary in an endemic area. Similar to some previous data, the prevalence of cholangiocarcinoma was slightly higher in males as compared to females [29, 30]. Other significant demographic data was a history of alcohol drinking and a family history of liver cancer in the cholangiocarcinoma cases as compared to non-cancer cohort, which could be important for the development of cholangiocarcinoma. In addition, we found a strong family history of cholangiocarcinoma in some patients. Cholangiocarcinoma patients had many first-degree relatives with liver cancer although most of them were diagnosed only by clinical suspicion, for example, obstructive jaundice with abdominal mass, mostly without histological confirmation. 
Although risk factors for the development of cholangiocarcinoma in the western countries are primary sclerosing cholangitis, an inflammatory disease of the biliary tract, and ulcerative colitis, certain parasitic liver diseases are notable risk factors in Asia. Colonization with the liver flukes O. viverrini (in Thailand, Laos and Vietnam) [3133] or Clonorchis sinensis (in China, Taiwan, Eastern Russia, Korea, and Vietnam) is associated with the development of cholangiocarcinoma [34]. In this study, we found that about $18-25 \%$ of our villagers had a history of liver flukes and 25-35\% of them had been treated with drugs to eradicate the parasites but no significant difference was observed between the cancer cases and the noncancer cases. There are many potential problems with detecting $O$. viverrini eggs by microscopic examination. For example, the egg features are similar to those of lecithodendrid and heterophyid parasites, therefore, the examination requires a high level of skill, and the methods are time consuming. To overcome these constraints, with the cooperation of the Department of Helminthology, Faculty of Tropical Medicine, Mahidol University, stool internal transcribed spacer (ITS)-PCR, which is a more sensitive and specific method, was performed in 22 cholangiocarcinoma patients during the first 2 years of the study [35]. All patients had positive results from stool PCR for $O$. viverrini, suggesting that $O$. viverrini continues to be an important contribution factor to the development of cholangiocarcinoma in Thailand.

Although we could detect early-stage disease and the majority of patients were able to receive curative surgery, the recurrence rate was still not as low as expected, necessitating our attempts to identify if tumor markers could be of better benefit than ultrasonography for early screening. Our study confirmed that currently available tumor markers have inadequate sensitivity and specificity for cholangiocarcinoma screening [36, 37]. These markers, such as CEA, CA 19-9, ALP, were not sensitive enough to detect early cholangiocarcinoma cases in our study cohort. Other new biological markers may be needed to identify patients at the earliest stage that will potentially lead to curative success $[35,38,39]$. Nevertheless, with the excellent outcome of our patients with premalignant lesions, we suggest that ultrasonography should be a first screening tool for cholangiocarcinomain an endemic area until other useful biological markers are discovered or become available. Studies are ongoing to identify new genomic and proteomic markers for cholangiocarcinoma cases in Thailand [40]. New therapeutic advances are also needed to improve the disease-free survival of patients undergoing surgical resection $[41,42]$.

\section{Conclusions}

This study represents the first large population-based screening program ever performed in an endemic area of cholangiocarcinoma by serial ultrasonography coupled with laboratory tests. The high prevalence and incidence rates of cholangiocarcinoma in Northern Thailand were evident. Current tumor markers and stool examination for parasites are not of benefit for cholangiocarcinoma screening in an endemic area. Ultrasonography should be offered as a first screening tool for cholangiocarcinoma in individuals aged $\geq 40$ in an endemic area. Patients with premalignant lesions achieved the most benefit from screening ultrasonography and are potentially cured. Future studies are needed to identify new biological markers that will capture cancer cells at the earliest stage of development.

\section{Abbreviations}

AFP, a-fetoprotein; ALP, alkaline phosphatase; CA 19-9, carbohydrate antigen 19-9; CBC, complete blood count; CCA, cholangiocarcinoma; CEA, carcinoembryonic antigen; CT, computed tomography; DFS, disease-free survival; $\mathrm{H} \& \mathrm{E}$, hematoxylin and eosin; ITS, internal transcribed spacer; IU, international unit; LFT, liver function tests; $\mathrm{mL}$, millilitre; $\mathrm{mm}$, millimeter; MRCP, magnetic resonance cholangiopancreatography; $\mathrm{MRI}$, magnetic resonance imaging; ng, nanogram; NPV, negative predictive value; O. viverrini, Opisthorchis viverrini; OS, overall survival; PCR, polymerase chain reaction; PFS, progression-free survival; PPV, positive predictive value; $U$, unit.

\section{Acknowledgments}

We appreciated all individuals, particularly the people of Nan Province, the staff of Chulabhorn Hospital, Nan Hospital and Ban Luang Hospital, including doctors, nurses, coordinators, the Nursing Division, and the Data Management team who generously spared their time for the accomplishment and fulfillment of this project. The excellent parasitic investigations by Faculty of Tropical Medicine, Mahidol University is also acknowledged.

\section{Funding}

The project is funded by Chulabhorn Hospital Research Grant (Certificate no. 29/2554).

\section{Availability of data and materials}

We do not wish to share all the data at the present time as the project is still ongoing. The full data set should be available by early spring of 2017 when the whole project is completed.

\section{Authors' contributions}

PS performed data collection, data analysis and manuscript drafting. SS, SV, AW and SS performed ultrasonography and imaging analysis. KA was responsible for subject recruitment, project coordination, and follow-ups of the cohort. TS reviewed and reported pathological results. RS and CB performed hepatic surgeries for the patients. SS and RM helped coordinating the appointments and follow-ups of the participants and patients. MY and GS were responsible for biospecimen collection and laboratory studies. KW and KS performed data management and statistical analysis. CA reviewed the study design, monitored the project, and critically revised the final manuscript. CM was responsible for the initiation and execution of the entire project. All authors read and approved the final manuscript.

\section{Competing interests}

The authors declare that they have no competing interests.

Consent for publication

Not applicable.

Ethics approval and consent to participate

This study was approved by the Ethics Committee for Human Research of Chulabhorn Research Institute, Bangkok, Thailand (Certificate no. 29/2554). Written informed consents were obtained from all the study participants using the Ethics Committee' approved forms. 


\section{Author details}

'Chulabhorn Hospital, 54 Kamphaeng Phet 6 Road, Laksi, Bangkok 10210, Thailand. 'Ban Luang Hospital, 191 Pa Kha Luang, Ban Luang, Nan 55190, Thailand. ${ }^{3}$ Faculty of Medicine Siriraj Hospital, Mahidol University, 2 Wanglang Road, Bangkoknoi, Bangkok 10700, Thailand. ${ }^{4}$ National Cancer Institute of Thailand, 268/1 Rama VI Road, Ratchathewi, Bangkok 10400, Thailand. ${ }^{5}$ Chulabhorn Research Institute, 54 Kamphaeng Phet 6 Road, Laksi, Bangkok 10210, Thailand.

\section{Received: 23 December 2015 Accepted: 26 May 2016}

Published online: 02 June 2016

\section{References}

1. Ustundag Y, Bayraktar Y. Cholangiocarcinoma: a compact review of the literature. World J Gastroenterol. 2008;14(42):6458-66.

2. Patel T. Increasing incidence and mortality of primary intrahepatic cholangiocarcinoma in the United States. Hepatology. 2001;33(6):1353-7.

3. West J, Wood H, Logan RF, Quinn M, Aithal GP. Trends in the incidence of primary liver and biliary tract cancers in England and Wales 1971-2001. Br J Cancer. 2006;94(11):1751-8.

4. Khan SA, Taylor-Robinson SD, Toledano MB, Beck A, Elliott P, Thomas HC. Changing international trends in mortality rates for liver, biliary and pancreatic tumours. J Hepatol. 2002;37(6):806-13.

5. Welzel TM, McGlynn KA, Hsing AW, O'Brien TR, Pfeiffer RM. Impact of classification of hilar cholangiocarcinomas (Klatskin tumors) on the incidence of intra- and extrahepatic cholangiocarcinoma in the United States. J Natl Cancer Inst. 2006;98(12):873-5.

6. McGlynn KA, Tarone RE, El-Serag HB. A comparison of trends in the incidence of hepatocellular carcinoma and intrahepatic cholangiocarcinoma in the United States. Cancer Epidemiol Biomarkers Prev. 2006;15(6):1198-203.

7. Malhi H, Gores GJ. Cholangiocarcinoma: Modern advances in understanding a deadly old disease. J Hepatol. 2006;45(6):856-67.

8. Blechacz BR, Gores GJ. Cholangiocarcinoma. Clin Liver Dis. 2008;12(1):131-50. ix

9. Thunyaharn N, Promthet S, Wiangnon S, Suwanrungruang K, Kamsa-ard S. Survival of cholangiocarcinoma patients in northeastern Thailand after supportive treatment. Asian Pac J Cancer Prev. 2013;14(11):7029-32.

10. Uttaravichien T, Bhudhisawasdi V, Pairojkul C, Pugkhem A. Intrahepatic cholangiocarcinoma in Thailand. J Hepatobiliary Pancreat Surg. 1999;6(2): 128-35.

11. Khuntikeo N, Pugkhem A, Titapun A, Bhudhisawasdi V. Surgical management of perihilar cholangiocarcinoma: a Khon Kaen experience. J Hepatobiliary Pancreat Sci. 2014;21(8):521-4.

12. Endo I, Gonen M, Yopp AC, Dalal KM, Zhou Q, Klimstra D, et al. Intrahepatic cholangiocarcinoma: rising frequency, improved survival, and determinants of outcome after resection. Ann Surg. 2008;248(1):84-96.

13. Mihalache F, Tantau M, Diaconu B, Acalovschi M. Survival and quality of life of cholangiocarcinoma patients: a prospective study over a 4 year period. J Gastrointestin Liver Dis. 2010;19(3):285-90

14. Shin HR, Oh JK, Masuyer E, Curado MP, Bouvard V, Fang YY, et al. Epidemiology of cholangiocarcinoma: an update focusing on risk factors. Cancer Sci. 2010;101(3):579-85.

15. Kamangar F, Dores GM, Anderson WF. Patterns of cancer incidence, mortality, and prevalence across five continents: defining priorities to reduce cancer disparities in different geographic regions of the world. J Clin Oncol. 2006;24(14):2137-50.

16. Jemal A, Bray F, Center MM, Ferlay J, Ward E, Forman D. Global cancer statistics. CA Cancer J Clin. 2011;61(2):69-90.

17. Vatanasapt V, Martin N, Sriplung H. Cancer in Thailand 1988-1991 (No. 16). Bangkok: IARC Technical Report; 1993. p. 88-9.

18. Brindley PJ, da Costa JM, Sripa B. Why does infection with some helminths cause cancer? Trends Cancer. 2015;1(3):174-82.

19. Sripa B, Pairojkul C. Cholangiocarcinoma: lessons from Thailand. Curr Opin Gastroenterol. 2008;24(3):349-56.

20. Siriarayapon $\mathrm{P}$, Taprasert $\mathrm{P}$, Kongyu S. Epidemiology of Liver Cancer in Banluang District, Nan Province. Weekly Epidemiol Surveillance Rep. 2006;37(3s).

21. Hakulinen T. Cancer Incidence in Five Continents. Vol. VII. Parkin DM, Whelan SL, Ferlay J, Raymond L and Young J. ed. IARC Scientific Publications No. 143, Lyon; 1997.

22. Egner JR. AJCC Cancer Staging Manual. JAMA. 304(15):1726-1727.
23. Hospital-Based Cancer Registry. National Cancer Institute. 2013. http://www. nci.go.th/th/File download/Nci\%20Cancer\%20Registry/HOSPITALBASED\%202013\%20All\%20(Online)\%20(1).pdf. Accessed 20 Mar 2016.

24. Maithel SK, Gamblin TC, Kamel I, Corona-Villalobos CP, Thomas M, Pawlik TM. Multidisciplinary approaches to intrahepatic cholangiocarcinoma. Cancer. 2013;119(22):3929-42.

25. Yusoff AR, Razak MM, Yoong BK, Vijeyasingam R, Siti ZM. Survival analysis of cholangiocarcinoma: a 10-year experience in Malaysia. World J Gastroenterol. 2012:18(5):458-65.

26. Nitta T, Nakanuma Y, Sato Y, Hirano S, Pairojkul C. Pathological characteristics of intraductal polypoid neoplasms of bile ducts in Thailand. Int J Clin Exp Pathol. 2015;8(7):8284-90.

27. Per Capita Income of Population by Region and Province: 1995-2013. Office of the National Economic and Social Development Board, Office of the Prime Minister. www.nesdb.go.th/. Accessed 25 Mar 2016.

28. Suzuki H, Isaji S, Pairojkul C, Uttaravichien T. Comparative clinicopathological study of resected intrahepatic cholangiocarcinoma in northeast Thailand and Japan. J Hepatobiliary Pancreat Surg. 2000;7(2):206-11.

29. Chang JS, Tsai CR, Chen LT. Medical risk factors associated with cholangiocarcinoma in Taiwan: a population-based case-control study. PLoS One. 2013;8(7), e69981.

30. Singal AK, Vauthey JN, Grady JJ, Stroehlein JR. Intra-hepatic cholangiocarcinoma-frequency and demographic patterns: thirty-year data from the M.D. Anderson Cancer Center. J Cancer Res Clin Oncol. 2011; 137(7):1071-8

31. Sripa B, Kaewkes S, Sithithaworn P, Mairiang E, Laha T, Smout M, et al. Liver fluke induces cholangiocarcinoma. PLoS Med. 2007;4(7), e201.

32. Watanapa P, Watanapa WB. Liver fluke-associated cholangiocarcinoma. Br J Surg. 2002;89(8):962-70.

33. Young ND, Nagarajan N, Lin SJ, Korhonen PK, Jex AR, Hall RS, et al. The Opisthorchis viverrini genome provides insights into life in the bile duct Nat Commun. 2014;5:4378

34. Hong ST, Fang Y. Clonorchis sinensis and clonorchiasis, an update. Parasitol Int. 2012;61(1):17-24.

35. Sato $M$, Pongvongsa $T$, Sanguankiat $S$, Yoonuan $T$, Dekumyoy $P$, Kalambaheti T, et al. Copro-DNA diagnosis of Opisthorchis viverrini and Haplorchis taichui infection in an endemic area of Lao PDR. Southeast Asian J Trop Med Public Health. 2010;41(1):28

36. Carpelan-Holmström M, Louhimo J, Stenman UH, Alfthan H, Haglund C. CEA, CA 19-9 and CA 72-4 improve the diagnostic accuracy in gastrointestinal cancers. Anticancer Res. 2001;22(4):2311-6.

37. Pungpak S, Akai P, Longenecker B, Ho M, Befus A, Bunnag D. Tumour markers in the detection of opisthorchiasis-associated cholangiocarcinoma. Trans R Soc Trop Med Hyg. 1991;85(2):277-9.

38. Yongvanit $\mathrm{P}$, Pinlaor $\mathrm{S}$, Loilome W. Risk biomarkers for assessment and chemoprevention of liver fluke-associated cholangiocarcinoma. J Hepatobiliary Pancreat Sci. 2014;21(5):309-15.

39. Ong CK, Subimerb C, Pairojkul C, Wongkham S, Cutcutache I, Yu W, et al. Exome sequencing of liver fluke-associated cholangiocarcinoma. Nat Genet. 2012;44(6):690-3.

40. Chaisaingmongkol J, Budhu A, Dang H, Rabibhadana S, Pupacdi B, Forgues $M$, et al. Abstract LB-173: The Thailand initiative in genomics and expression research for liver cancer (TIGER-LC): Defining novel subtypes of hepatocellular carcinoma and cholangiocarcinoma. Cancer Res. 2015;75(15 Supplement):LB-173.

41. Kabbach G, Assi HA, Bolotin G, Schuster M, Lee HJ, Tadros M. Hepatobiliary tumors: update on diagnosis and management. J Clin Transl Hepatol. 2015; 3(3):169-81

42. Rizvi S, Borad MJ, Patel T, Gores GJ. Cholangiocarcinoma: molecular pathways and therapeutic opportunities. Semin Liver Dis. 2014;34(4):456-64. 